\title{
A Mobile Robot Teleoperation System with a Wireless Communication Infrastructure using a Leaky Coaxial Cable based on TCP/IP
}

\author{
Kei Sawai ${ }^{1}$, Satoshi Aoyama ${ }^{2}$, Tatsuo Motoyoshi ${ }^{3}$, Toru Oshima ${ }^{4}$, Ken'ichi Koyanagi $^{5}$ \\ Hiroyuki Masuta ${ }^{6}$, Takumi Tamamoto ${ }^{7}$ \\ Department of Intelligent Robotics \\ Toyama Prefectural University \\ Toyama, Japan
}

\begin{abstract}
In this study, we propose and develop a wireless teleoperation system for mobile robots using a leaky coaxial cable (LCX) with a wireless communication infrastructure. In closed spaces resulting from disasters, some problems have been reported, such as cable entanglement, disconnection of wired communications, and problems with teleoperations (e.g., unstable communication quality for wireless communications). In this paper, we propose a communication infrastructure system for teleoperation of a mobile robot using LCXs as a communication infrastructure that considers the above issues. In addition, the communication quality was measured for the operability of the mobile robot by constructing an IEEE $802.11 \mathrm{~b} / \mathrm{g} / \mathrm{n}$ network using an LCX, and the effectiveness of the proposed system in an actual environment was confirmed. In the evaluation of the communication quality, bandwidth compression throughput values and packet jitter were measured as evaluation items at the packet level to objectively consider the teleoperation controllability.
\end{abstract}

Keywords-Mobile robot; teleoperation; leaky coaxial cable; teleoperation infrastructure

\section{INTRODUCTION}

After a disaster occurs, disaster reduction activities that minimize damage in the disaster-stricken area are important for early recovery and reconstruction activities [1]. In the Great Hanshin-Awaji Earthquake, survivors buried in the rubble had a survival rate of less than $5 \%$ after $72 \mathrm{~h} \mathrm{[2-3].} \mathrm{In} \mathrm{order} \mathrm{to} \mathrm{carry}$ out rescue activities quickly and effectively, it was necessary to perform disaster mitigation activities. Disaster mitigation is a method to minimize damage after a disaster occurs [4]. It is important to collect information on the disaster-stricken area during these disaster mitigation activities, where drones and already existing communication infrastructures are often used. However, in closed spaces, it is impossible to collect information from the sky, and the risk of failure of the already existing infrastructure is high. Therefore, these methods are difficult to use. In addition, information gathering by rescue workers is known to create a high risk of secondary disasters, such as fires and collapses, in closed spaces [5].

Therefore, considering these problems, various methods used to gather information via multirobot systems have been studied to reduce the secondary disaster risk caused by rescue workers.
The communication methods used by mobile robots include wired communication and wireless communication. The teleoperator of a mobile robot needs to use different communication methods according to the situation in the disaster-stricken area. Wired communication provides highquality communication between the teleoperator and the mobile robot because this method has the advantage of being able to supply power from a cable. However, when operating a multirobot, there are physical limitations, such as the entanglement of the cables of the mobile robot. Therefore, in multirobot operations involving wired communication, one problem is the depression of the running performance of the multirobot and the disruption of communication owing to cable disconnection.

However, communication using radio waves can achieve teleoperations while maintaining the running performance of the mobile robot. However, when the mobile robot moves behind a wall or an obstacle, radio waves can get blocked; hence, increasing the risk of disconnection between the teleoperator and the mobile robot [6]. Additionally, radio waves are prone to attenuation; thus, the teleoperation range of mobile robots is smaller for wireless communication compared to wired communication. When the tele-operator controls a mobile robot, it is important to determine the best teleoperation method needed according to the environment of the disasterstricken area; however, it is difficult to predict the situation of a disaster-stricken area in advance. Therefore, it is important to discuss various teleoperation methods that can be used in disaster-stricken areas.

We discussed using multirobot teleoperation systems in closed spaces that use leaked radio waves from leaky coaxial cables (LCXs) [7]. The purpose of this study was to develop a teleoperation method that is resistant to cable cutting and entanglement using an LCX while maintaining the mobile performance of the mobile robot. This is a new teleoperation method developed for closed disaster-stricken spaces, and it can promote disaster reduction activities from the viewpoint of diversification of teleoperation methods. However, teleoperation studies that involve LCXs have not yet defined the standardized operation method for mobile robots. In this paper, we propose a teleoperation method for a mobile robot 
that uses LCXs with TCP/IP, and we evaluate the operability of the teleoperation of the mobile robot at the TCP/IP level.

In Section II, we have presented the problems which Wireless Teleoperation of a Mobile Robot in Closed DisasterStricken Spaces. Section III describes mobile robot system using an LCX as the communication infrastructure. Section IV describes throughput and packet jitter measurement methods to verify the teleoperation performance of the LCX. Section V has evaluated our proposed method which is maintaining the controllability of the mobile robot teleoperation using a TCP/IP-Compliant LCX network. Sections VI and VII has the discussion and conclusion.

\section{WiRELESS TELEOPERATION OF A MOBILE ROBOT IN ClOSED DisAster-STRICKEN SPACES}

\section{A. Wireless Teleoperation using ad hoc Networks}

Many wireless teleoperation systems for mobile robots are based on TCP/IP protocols. The Robot Wireless Sensor Network (RWSN) is one method that involves the teleoperation of mobile robots using wireless communication [8-20]. RWSN is a system that provides information on a disaster, which can expand the communication distance between remote operators and mobile robots. In this system, while the mobile robot moves in the disaster-stricken area, the sensor node ( $\mathrm{SN})$, which is a small wireless computer, is deployed on the movement route to expand the wireless teleoperation range (Fig. 1). Then, most of the communication between the remote operator and the mobile robot conforms to $\mathrm{TCP} / \mathrm{IP}$ protocols, in which the teleoperation is realized via packet communication. TCP/IP is highly compatible with the communication of the mobile robot because most of the control system of a mobile robot involves a PC. Therefore, socket communication is often adopted for mobile robot communication, and information communication by packet transmission and reception is typical. Therefore, in our system, we adopted a wireless LAN as the communication method.

After SNs are deployed, a wireless connection is made at each SN to construct a bead-like wireless ad hoc network. Using the RWSN, the remote operator can transmit while relaying instructions to the mobile robot. Therefore, the remote operator can gather information using the mobile robot while expanding the operational range. Moreover, in the RWSN, each SN can share the acquired information, and it is easy to expand and repair the network using a mobile robot. Therefore, this system is effective for collecting information in places where it is difficult to build a communication infrastructure and in disaster-stricken areas where there is a high risk of communication infrastructure disruption. However, in a beaded wireless ad hoc network, the problem is that the transmission speed decreases as the number of relays increases. Therefore, teleoperation of a mobile robot using an ad hoc network is difficult to operate in an environment with many corners that require many relays.

Additionally, network disconnection owing to the physical failure of the SN can make it difficult for the mobile robot to return.

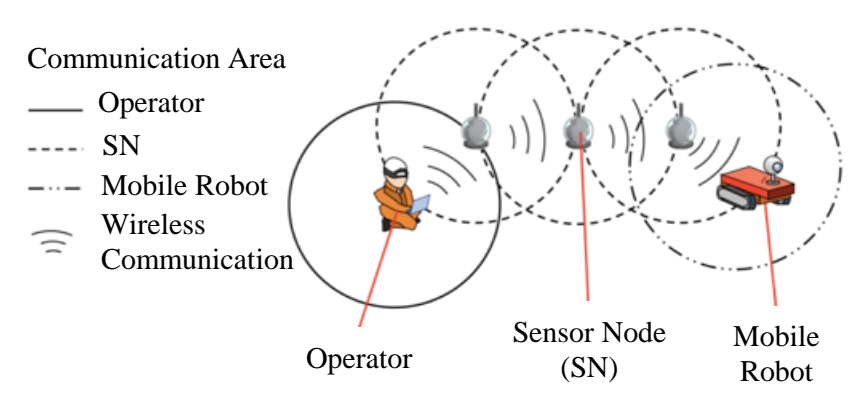

Fig. 1. RWSN.

\section{B. Considerations for Wireless Teleoperation of a Mobile Robot}

If the communication quality of the communication path is poor, the mobile robot may be disconnected from the network. Additionally, when the communication quality becomes unstable, the sensing information collected by the mobile robot cannot be transmitted to the remote operator; thus, the risk to the operability of the teleoperation is increased.

Thus, the communication infrastructure used for a new mobile robot teleoperation method requires a communication standard that provides easy monitoring of the communication quality and stable control. Additionally, when constructing a multirobot environment using the RWSN, routing becomes complicated, the number of relays increases, and it becomes difficult to maintain the transmission speed required for the operation of a mobile robot. Therefore, it is difficult to use the RWSN to gather information using a multirobot system. Regarding the movement distance, if the distance is extended too much, there will be a risk that the mobile robot will not be able to return if the relay is damaged. Considering utilization in affected areas, the fault tolerance of the communication equipment should be discussed as well as the distance extension. Therefore, for remotely controlling a mobile robot in an enclosed space in a disaster-stricken area, it is important to propose a new method that considers the following points.

- Adopting a wireless communication method that is capable of implementing a TCP/IP protocol.

- Communication quality maintenance in a multirobot environment.

- Teleoperation in large spaces.

- Network construction using communication equipment that is resistant to physical damage.

In the next section, we will consider a wireless teleoperation method for a mobile robot using an LCX, which considers the above points.

\section{MobiLe Robot System USING AN LCX AS THE COMMUNICATION INFRASTRUCTURE}

\section{A. Teleoperation System using an LCX}

As mentioned in Section II, in order to develop a new teleoperation system for a mobile robot for use in a damaged closed space, it is necessary to consider the problems of the RWSN. In the previous section, we clarified the issues that 
need to be considered and the requirements for the teleoperation of mobile robots in a closed space. Taking these into consideration, we have developed a wireless teleoperation method for mobile robots using an LCX. Because LCXs enable long-distance communication and network construction while maintaining high-quality communication, teleoperation can be performed while maintaining the traveling performance and communication quality of the mobile robot. Furthermore, LCXs create a wireless network around the cable by leaking radio waves from the cable. Moreover, when the LCXs are physically disconnected, communication up to the disconnection point is possible, and the specification for physical failure is strong.

Therefore, it is possible to construct a multirobot environment that is wirelessly connected using a single wired communication cable. However, LCXs have not been studied for teleoperation based on TCP/IP for mobile robots. In this paper, we propose a communication infrastructure that uses an LCX for TCP/IP as a mobile robot teleoperation system. Moreover, we evaluate the communication quality between the mobile robot and the remote operator at the packet level and discuss the teleoperation of the mobile robot on the basis of these results.

\section{B. Communication Characteristics of the $L C X$}

LCXs (Fig. 2(a)) have been studied and developed for fire radio and radio broadcasting in closed spaces, such as tunnels and subways. LCX is a cable designed to periodically make holes called "SLITS" in the outer conductor of a coaxial cable and leak part of the electrical signal from the "SLITS" to the outside (Fig. 2(b)). Information transmitted by the remote operator to the mobile robot is transmitted around the LCX by leaking radio waves from the SLITS. When the LCX is connected to an access point, the entire cable can be used as one antenna. The communication environment can be expanded in a wide area along the cable by laying the LCX, and a multirobot environment can be constructed. Even if the LCX is disconnected at some location along the path, it is possible to construct a network between the access point and its broken part.

Fig. 3 shows a system for gathering information from a disaster-stricken area using multi-robots with an LCX as a wireless communication infrastructure. This system assumes use in closed spaces after a disaster. In this system, after a disaster occurs, a cable-laying robot equipped with an LCX uses the radio waves leaked from the LCX and lays out the LCX while advancing in the closed space. After the LCX is laid out, the mobile robot becomes able to perform longdistance communication without physical restriction from the wired cable using the radio waves leaked from the LCX.

\section{Communication Infrastructure for Teleoperation using LCX Networks with TCP/IP Protocols}

The communication method used in our proposed system included a wireless LAN from the IEEE 802.11 series. Currently, general-purpose PCs are generally used as the control systems for mobile robots. Moreover, communication between general-purpose PCs is highly compatible with TCP/IP for performing flexible network construction. Therefore, in addition to the possibility of packet communication, communication between the remote operator and the mobile robot needs to adopt a communication standard that can work with the control system of the mobile robot.

Thus, for this system, we decided to use the IEEE 802.11 series as the communication protocol. Then, we adopted IEEE $802.11 \mathrm{~g}$ for network connection between the LCX and the mobile robot. Throughput, which is the information transmission rate of IEEE $802.11 \mathrm{~g}$, has a theoretical value of $54.0 \mathrm{Mbps}$. Nitta et al. studied the wireless teleoperation of heavy-equipment robots while streaming camera images and reported that teleoperation is possible if the throughput value is 4.0 Mbps or more [8]. Additionally, in our proposed system, teleoperation is possible if the communication speed between the remote operator and the mobile robot is $4.0 \mathrm{Mbps}$ or more, and IEEE $802.11 \mathrm{~g}$ meets this requirement. In addition, teleoperation of multi-robots using an LCX as a communication infrastructure is required to maintain a certain operation performance level by the packet control.

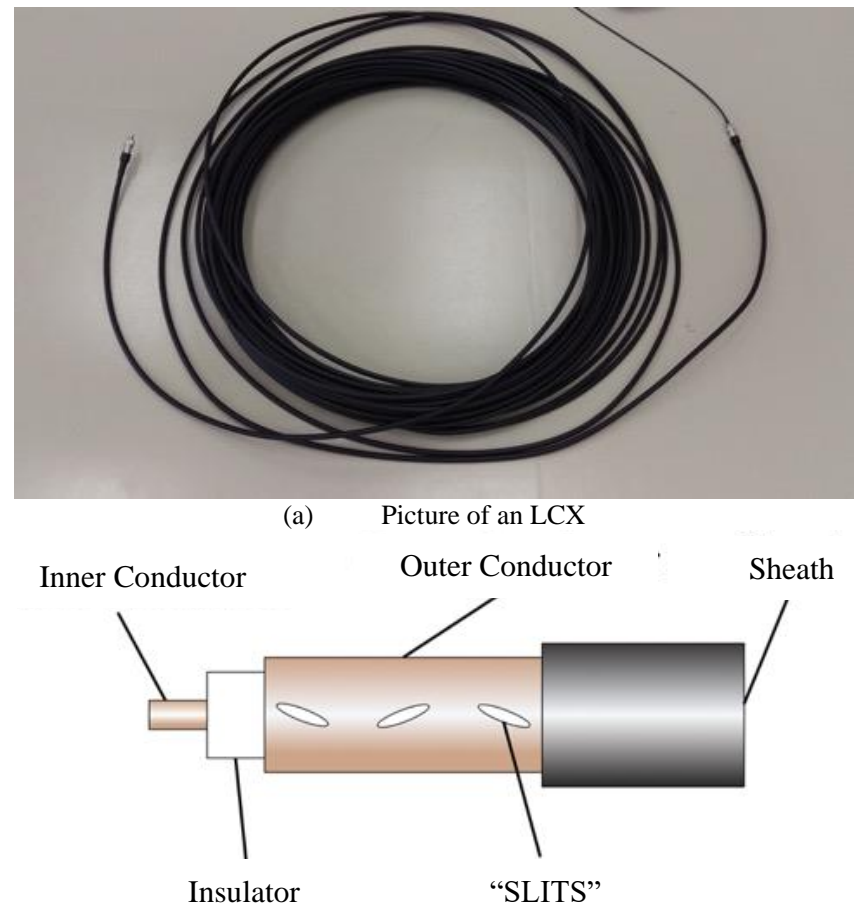

(b) Internal structure of the cable

Fig. 2. LCX.

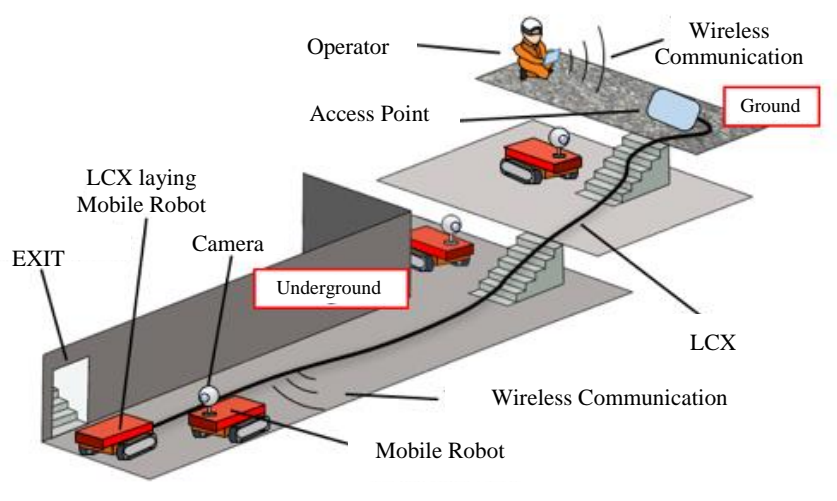

Fig. 3. Teleoperation System of a Mobile Robot using an LCX. 


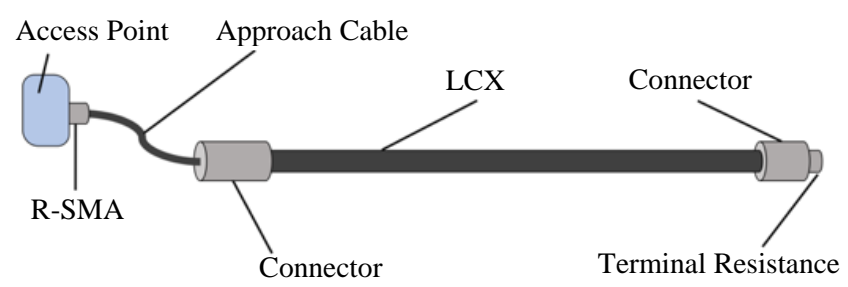

Fig. 4. An LCX Connected to an Access Point Capable of TCP/IPCompliant Network Construction.

Therefore, we decided to use UDP communication without confirming the connection response request as the communication standard for packet communication between the multirobot and the operator. In our network construction based on TCP/IP, we used an access point with an SMA terminal that can connect to the LCX (Fig. 4).

In the next section, communication quality properties that need to be evaluated between the mobile robot and the remote operator will be defined, and the methods for measuring them will be described. In addition, we built a TCP/IP-compliant network using an LCX, and we will discuss the evaluation of the communication quality at the packet level.

\section{ThroughPUt AND PACKET JitTER MEASUREMENT METHODS TO VERIFY THE TELEOPERATION PERFORMANCE OF THE LCX}

\section{A. Evaluation of the Transmission Speed}

Remote operators can operate by receiving images and sensor information acquired by a mobile robot. However, because the amount of information fluctuates, it is necessary to monitor the communication quality to maintain a stable operation. Therefore, it is necessary to determine the transmission speed between the operator and the mobile robot during teleoperation. The transmission speed on the TCP/IPcompliant communication path was evaluated as the throughput at the packet level. Throughput [bps] indicates the amount of transmission per second received by the receiving computer (Fig. 5).

Additionally, bandwidth compression throughput represents the maximum receivable number per second by transmitting a large number of packets from the transmitting side. It is assumed that a large amount of information will be transmitted for the information acquired by the mobile robot in a disaster-stricken area, such as three-dimensional space information, moving image information, and different types of sensing information. Therefore, for the teleoperation of a mobile robot via an LCX, we used a band compression throughput measurement that can determine the upper limit of the transmission speed. Additionally, in our system, to measure the accurate upper limit of the throughput, we sent as many packets as the computer could process. The packet size of the transmission packet was set to 1,400 bytes, which is the upper limit. In this system, the bandwidth compression throughput was calculated assuming that the packet size is $B$ [byte], the total number of received packets is $n$, and the time required to complete the measurement is $t[\mathrm{~s}]$. Then, the band compression throughput, $T h$ [bps], can be expressed by the following equation:

$T h=\frac{8 B n}{t}$

The reduction in the upper limit of throughput delays the transmission and reception of sensing information and deteriorates the operability of a mobile robot. Therefore, it is important to monitor the bandwidth compression throughput when the mobile robot is remotely operated. Therefore, we developed an application for evaluating the bandwidth compression throughput and implemented it on the mobile robot and the teleoperation computer.

\section{B. Evaluation of Packet Fitter}

A remote operator sends command packets to teleoperate a mobile robot. In order to maintain the operability of the mobile robot, it is necessary to send and receive command packets at fixed time intervals. Disturbances in the transmission and reception interval of the command packets reduce operability because they interfere with teleoperations. Therefore, it is necessary to monitor the transmitted and received intervals to maintain operability. Therefore, in our proposed system, we evaluated the packet jitter to monitor the transmission and reception intervals of the command packets. Packet jitter is a disturbance in the arrival interval of packets. Assuming (1) 2 packet transmission interval is $T \mathrm{~ms}$ and the deviation us ure reception interval is $d \mathrm{~ms}$, the reception fluctuation is $T+d$ ms. A schematic of packet jitter is shown in Fig. 6.

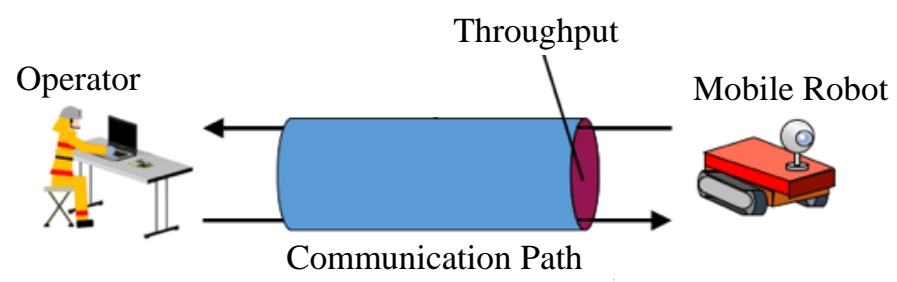

Fig. 5. Throughput [bps], the Amount of Information Transmitted Per unit Time.

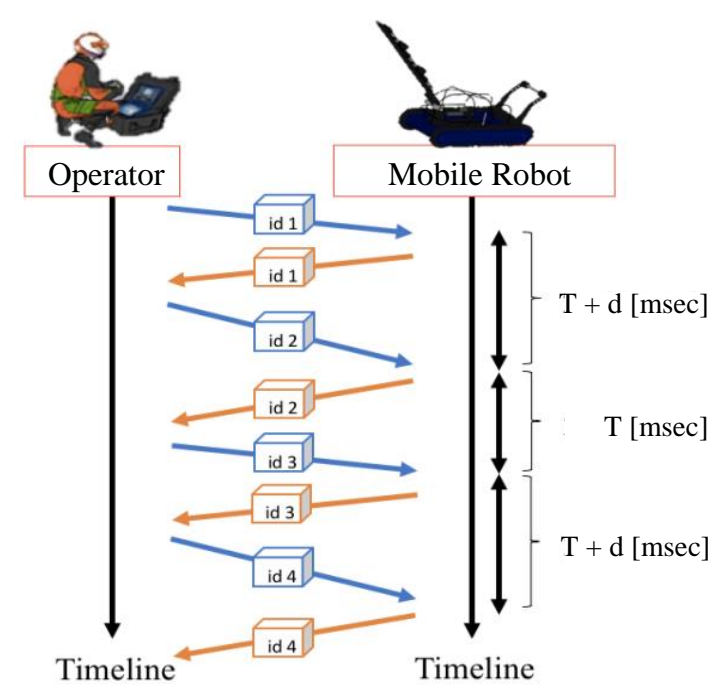

Fig. 6. Packet Jitter [ms], Fluctuations in the Packet Arrival Time. 
Disturbances in the packet arrival intervals affect real-time applications. In VoIP and video streaming, disturbances in the packet arrival interval cause interruptions in the audio and the deterioration of the smoothness of the video. Even in the teleoperation of mobile robots, it is important to consider packet jitter because it is the same real-time system as these applications. In our proposed system, the command packet transmission interval was set to the same $20 \mathrm{~ms}$ interval as in real-time applications, such as VoIP and Skype. Remote operations are not affected when the packet reception range is 19-21 ms. However, arrival of packets outside this range must be monitored because of the problems mentioned above. Therefore, we developed an application for packet jitter evaluation and implemented it on a mobile robot and a teleoperation computer.

In the next section, we will discuss the operability of teleoperation of a mobile robot using a network constructed using an LCX with TCP/IP. In our evaluation experiment, we constructed the network in a real environment and evaluated the band compression throughput and packet jitter to determine the operability of the teleoperation of the mobile robot when using an LCX and determined the effectiveness at the packet level.

\section{Controllability of the Mobile Robot TELEOPERATION USING A TCP/IP-COMPLIANT LCX NETWORK}

\section{A. Evaluation of Teleoperation Ability}

In this experiment, an LCX was laid in a straight line and a wireless LAN environment was built around the LCX. Then, a computer for teleoperation and a mobile robot were wirelessly connected to the network built by the LCX, and the mobile robot was moved away from the operator to measure the communication quality. In this experiment, bandwidth compression throughput and packet jitter were measured as indices to evaluate the operability of the teleoperation. The mobile robot received a command packet containing movement information from the operator and was set to move according to the information in the command packet. In order to evaluate the bandwidth compression throughput, the number of packets used for transmission and reception was set to 10,000 to provide an accurate evaluation. In addition, for the evaluation of packet jitter, we transmitted 500 (packet size: 200 bytes) packets, taking into consideration the influence of various noises from the experimental environment.

\section{B. LCX Network Connection Configuration}

Fig. 7 shows the network configuration for this experiment. The TCP/IP-compliant access point was wired to the LCX, and a wireless LAN environment (IEEE 802.11g) was constructed around the LCX. The laying range of the LCX was a straight line of $100 \mathrm{~m}$, and the wireless LAN network was developed in that range. For communication between the mobile robot and the remote operator, UDP was adopted by considering teleoperation, which is a real-time application.

The computer used for the teleoperation and the mobile robot were set to a static IP address to use this system in a multirobot environment. The network configuration shown in Fig. 3 looks like a bus-type topology, but this network topology was set up with a star-type topology for an easy-toconnect setup.
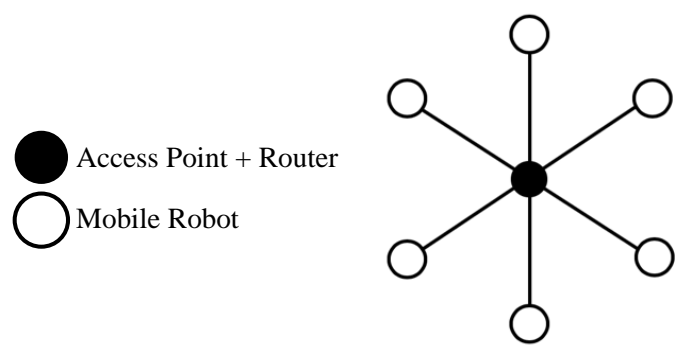

Fig. 7. Wireless Network and Mobile Robot Network Topology Constructed using an LCX (in the Experiment, only one Mobile Robot was Connected).

\section{Network Construction Area Constructed by an LCX}

In the teleoperation of mobile robots, it is necessary to determine in advance the construction range of the network used as the wireless communication infrastructure. The network construction area using an LCX as the communication infrastructure is the surrounding environment of the LCX in which the LCX is placed. However, for the teleoperation of our mobile robot, an area with a throughput of $4.0 \mathrm{Mbps}$ or more was used as the network construction area, and it was necessary to evaluate it at the packet level.

For the throughput measurement, the bandwidth was compressed by transmitting a large number of packets on the communication path, and the calculation was performed by counting the number of packets that could be transmitted and received per unit time. This was measured by connecting a remote operator terminal to an access point that constructed a wireless LAN using a wire and by measuring the throughput between the LCX and a computer terminal that was connected wirelessly. For evaluation, a $100 \mathrm{~m} \mathrm{LCX}$ was wired to the access point, laid in a straight line. The measurement was performed by moving the computer terminal in increments of $10 \mathrm{~m}$, and the measurement was performed five times to obtain an average value. The computer terminal was placed at a distance of $0.3 \mathrm{~m}$ vertically from the LCX.

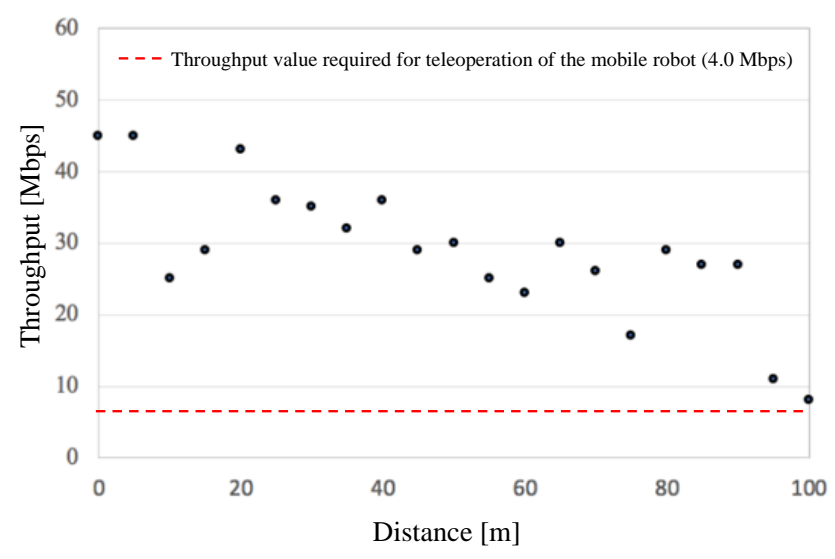

Fig. 8. Evaluation Results of the Network Construction Range of the LCX as a Function of the Throughput. 
Evaluation experiments were conducted in an environment where no other wireless LANs existed. The network construction area when using the LCX was evaluated by the throughput measurement shown in Fig. 8. In this experiment, it was confirmed that the network constructed by the LCX used in the evaluation experiment for the proposed method could obtain a throughput value of $4.0 \mathrm{Mbps}$ or more in the entire range. Throughput values tend to decrease as the distance from the Access Point increases; however, this is due to attenuation because of the cable length and is a normal result.

\section{Movement of the Mobile Robot during a Communication Quality Measurement}

The mobile robot moved remotely from the access point installation side $(0 \mathrm{~m})$ to the end point of the LCX $(100 \mathrm{~m})$ (Fig. 9). The movement path of the mobile robot was set $0.3 \mathrm{~m}$ away from the LCX in the vertical direction and parallel to the LCX. This experiment was conducted on a straight path of $100 \mathrm{~m}$ where no other wireless LAN environments existed (Fig. 10). Throughput and packet jitter were obtained by stopping the mobile robot at intervals of $5 \mathrm{~m}$ from $0 \mathrm{~m}$ to $100 \mathrm{~m}$ and by measuring five times to obtain the average value. Additionally, during the measurement of the communication quality, all communications related to teleoperation were stopped to provide an accurate result.

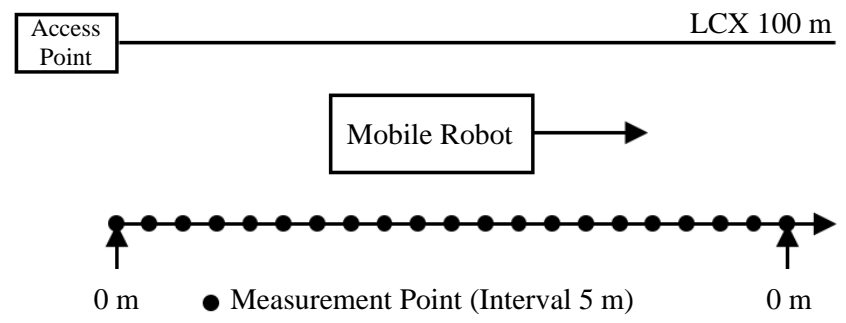

Fig. 9. Measurement Points of Communication Quality.
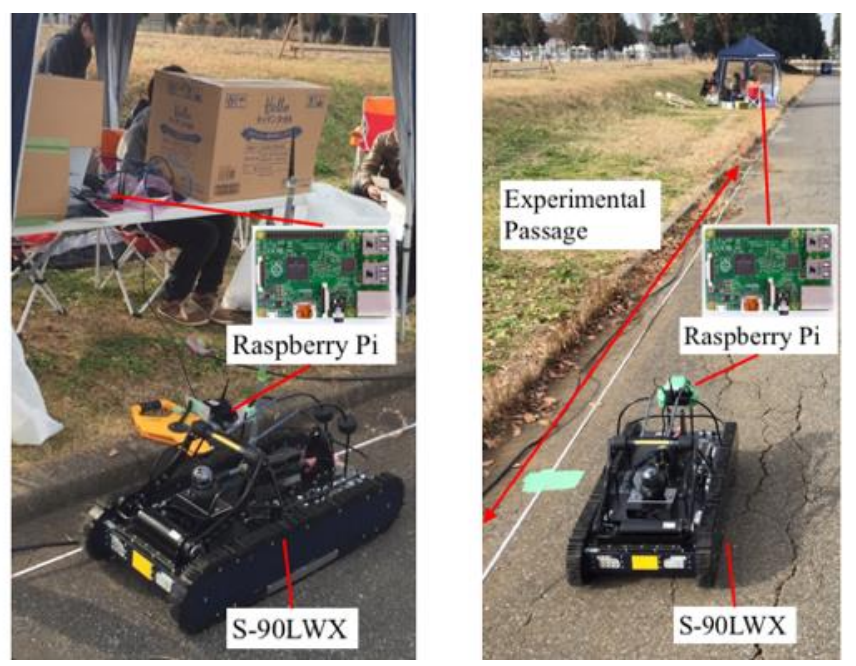

Fig. 10. Experimental Environment.

\section{E. Equipment used for Teleoperation Experiments of the Mobile Robot}

Raspberry Pi 2 (Model B) was used for the teleoperation computer and for the control system on the mobile robot. The LCX cable adopted was an LCXF-8D-LCX (HC Networks, Ltd., Tokyo, Japan), and the wireless access point used was AP-214 (Aruba Networks, Ltd., Santa Clara, CA, USA). For the mobile robot, S-90LWX (Topy Industries, Ltd., Tokyo, Japan) equipped with a crawler for the drive system was used as the rescue robot. We used the same Raspberry Pi 2 (Model B) as the remote control computer for the control system of the mobile robot, and adopted IEEE $802.11 \mathrm{~g}$ for the communication system.

\section{F. Results of Throughput and Packet Jitter in the Teleoperation Experiment of the Mobile Robot using the LCX Network}

Fig. 11 shows the results of the evaluation of the throughput. According to our results, the mobile robot was able to move up to $100 \mathrm{~m}$. The throughput value was maintained at 4.0 Mbps or higher, which is required for the teleoperation of the mobile robot, in the interval of $0-95 \mathrm{~m}$. The average result at all measurement points was $25.7 \mathrm{Mbps}$, which was a value that would not affect the remote control of the mobile robot. However, the throughput value at $10 \mathrm{~m}$ was $33.3 \mathrm{Mbps}$ lower than the value at $5 \mathrm{~m}$. The result at $75 \mathrm{~m}$ was $17.4 \mathrm{Mbps}$ lower than that at $70 \mathrm{~m}$. The results for the $100 \mathrm{~m}$ point could not be measured because the network connection was disrupted. However, because the mobile robot was able to move up to $100 \mathrm{~m}$, the communication quality was maintained just before the $100 \mathrm{~m}$ point.

Fig. 12 shows the results of the evaluation of packet jitter. We evaluated the proportion of the number of packets with a packet arrival interval of 19-21 ms. The results show that the ratio of the number of packets for the packet arrival interval of $19-21 \mathrm{~ms}$ was over $70 \%$ in the $0-95 \mathrm{~m}$ section, and the operability of the teleoperation did not decrease. However, the evaluation results at $100 \mathrm{~m}$ could not be obtained owing to network disconnection.

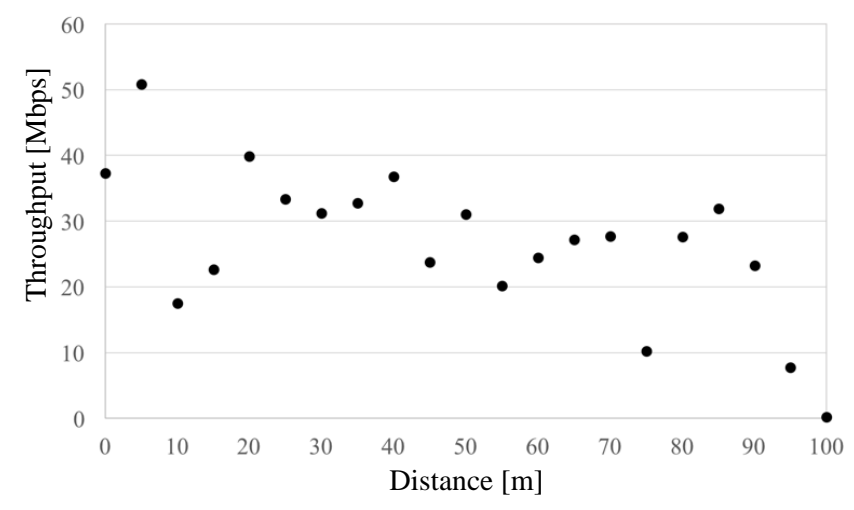

Fig. 11. Results of the Throughput Measurement. 


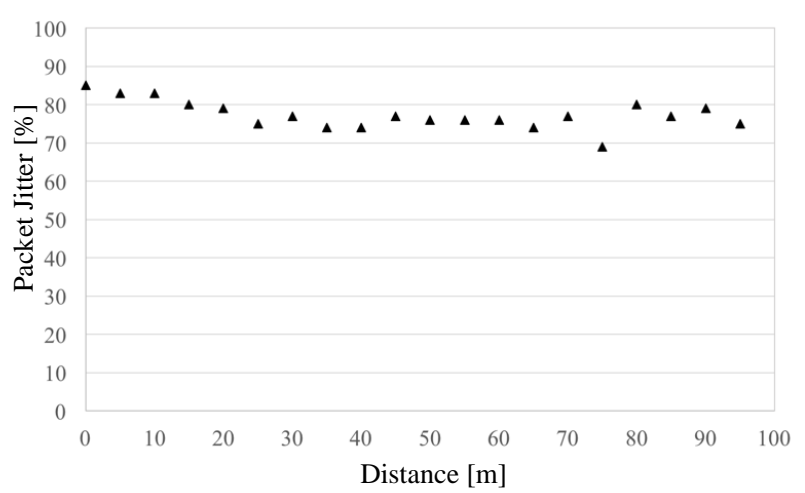

Fig. 12. Results of the Packet Jitter Measurement.

From these results, we confirmed that the teleoperation of a mobile robot using a TCP/IP-compliant LCX network can be performed without operability degradation or failure of realtime applications.

\section{DISCUSSION}

For evaluating the communication quality of the LCX network, throughput and packet jitter were measured in the range of $0-95 \mathrm{~m}$. In addition, it was confirmed from the evaluation results that the values required for teleoperation can be obtained at all the measurement points except for the $100 \mathrm{~m}$ point. Therefore, the teleoperation range of the mobile robot can be constructed within the range of $0-95 \mathrm{~m}$. The throughput decreased by $10.0 \mathrm{Mbps}$ or more at measurement points of 10 , 45,55 , and $75 \mathrm{~m}$ compared to that at measurement points immediately prior to these. The result was higher than 4.0 Mbps and did not affect the teleoperation of the mobile robot. At the next measurement point following the decreased measurement point, the throughput increased and recovered. The reason for these fluctuations may be a decrease in throughput due to environmental noises at the measurement point. Additionally, at the $95 \mathrm{~m}$ measurement point, the value was less than $10.0 \mathrm{Mbps}$ and could not be measured at $100 \mathrm{~m}$. The radio waves of LCXs have directivity on the access point side as shown in Fig. 13 and disconnection of the communication at the $100 \mathrm{~m}$ point may have occurred because the antenna of the mobile robot was out of the network.

For the packet jitter result, a packet reception rate of $70 \%$ or higher was observed except at the $75 \mathrm{~m}$ measurement point. At $75 \mathrm{~m}$, the measured throughput also significantly decreased. This may have been due to environmental noises; however, because the reception rate was $69 \%$, teleoperation was possible. At the next $80 \mathrm{~m}$ measurement point, the packet jitter became $80 \%$ and recovered.

This stability in the communication quality is a feature of communication using an LCX and is difficult to realize by a radio relay system, such as an RWSN. It is effective for use as an infrastructure for teleoperation in a disaster-stricken area where there is a high possibility of local environmental noise. In addition, there was no network disconnection or inoperable state between the operator and the mobile robot at $0-95 \mathrm{~m}$ of teleoperation.

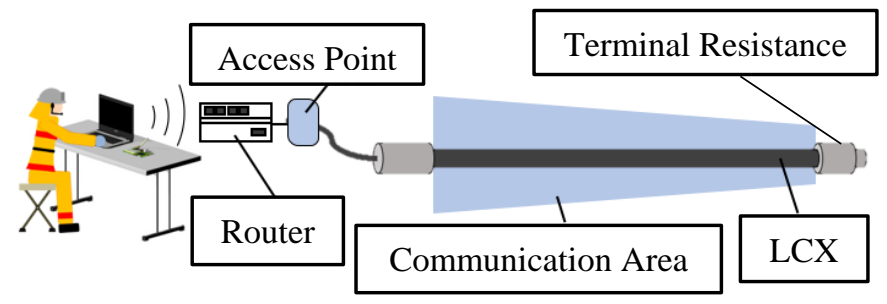

Fig. 13. Wireless Communication Area of the LCX because there was Directivity on the Access Point side, the Communication Range became Narrower as it Got Closer to the Terminal Resistance Side.

\section{VII.CONCLUSION}

In this paper, we proposed a new teleoperation system that implements TCP/IP on an LCX as a teleoperation method for mobile robots in a closed space. The LCX can construct a wireless network around the cable by emitting radio waves from the cable. By implementing TCP/IP on an LCX, and can effectively operate a mobile robot. Wired and wireless communication systems have been used to operate mobile robots in closed disaster-stricken spaces. However, physical disconnection and entanglement of cables and destabilization of communication quality in wireless communications are issues that hinder multirobot system deployment in closed spaces. The proposed system uses only one cable in a multirobot system; thus, the risk of physical disconnection and tangling is reduced. Moreover, in this system, the evaluation experiment confirmed that it is possible to maintain the communication quality between the mobile robot and the remote operator by combining wired communication with wireless communication. We adopted TCP/IP, which is widely used for robot communication as a communication standard, and it is a specification that can be implemented on many mobile robots. In our evaluation experiment, the effectiveness of the proposed method was confirmed using a mobile robot, and the operator was able to operate it without any operability issues during the experiment. Therefore, this proposed system can be effective as a new teleoperation method for mobile robot use in closed spaces after disasters.

This system is effective for multirobot system operations; however, in the future, we will implement the Resource Reservation Protocol for communication bandwidth compression of LCXs for effective operation in disasterstricken areas.

\section{REFERENCES}

[1] CHI Hao-yuan, LIU Xu, XU Xiao-dong: A Framework for Earthquake Disaster Mitigation System, Proceedings of 2011 China located International Conference on Information Systems for Crisis Response and Management (ISCRAM), pp.490-495, 2011.

[2] Y. Kawata: The great Hanshin-Awaji earthquake disaster: damage, social response, and recovery, Journal of Natural Disaster Science, Vol. 17, No. 2, pp.1-12, 1995.

[3] Y. Kawata: Disaster Mitigation due to next Nankai earthquake tsunami occurring in around 2035, Proc. of International Tsunami Symposium 2001, session 1, pp. 315-329, 2001.

[4] Y. Kawata: The great Hanshin-Awaji earthquake disaster: damage, social response, and recovery, Journal of Natural Disaster Science, Vol. 17, No. 2, pp.1-12, 1995. 
[5] Abishek T K, Chithra K R and Maneesha V. Ramesh: ADEN: Adaptive Energy Efficient Network of Flying Robots Monitoring over Disaster Hit Area, Proc. of 8th IEEE International Conference on Distributed Computing in Sensor Systems (IEEE DCOSS), pp.306-310, 2012.

[6] Abishek T K, Chithra K R, Maneesha V Ramesh: AER: Adaptive Energy Efficient Routing Protocol For Network of Flying Robots Monitoring over Disaster Hit Area, Proc. of 21st Annual Wireless and Optical Communications Conference (WOCC), pp.166- 169, 2012.

[7] S. Aoyama, K. Sawai, T. Oshima, K. Koyanagi, T. Motoyoshi, Hiroyuki Masuta and Takumi Tamamoto,: Proposal of Disconnection Prevention System in Multi-Robot System Utilizing LCX Network by Monitoring Broadcast Packets Storing Traffic Information, Proc. of the 3rd Fukushima Research Conference on Remote Technologies for Nuclear Facilities 2018, p. 18, 2018.

[8] T. Suzuki, K. Sawai, H. Kono and S. Tanabe: Sensor Network Deployment by Dropping and Throwing Sensor Node to Gather Information Underground Spaces in a Post-Disaster Environment, Descrete Event Robot, iConcept PRESS, ISBN 978-1-922227-05-8, 2012, in press.

[9] K. Sawai, H. Kono, S. Tanabe, K. Kawabata, T. Suzuki: Design and Development of Impact Resistance Sensor Node for Launch Deployment into Closed Area, In international journal of sensing for industry(Sensor Review), Emerald Group Publishing Ltd., Vol. 32, pp.318-326, 2012.

[10] S. Tanabe, K. Sawai and T. Suzuki: Sensor Node Deployment Strategy for Maintaining Wireless Sensor Network Communication Connectivity, International Journal of Advanced Computer Science and Applications (IJACSA), The Science and Information organization, Vol.2, No. 12, pp.140-146, 2011.

[11] Y. Koike, K. Sawai and T. Suzuki: A Study of Routing Path Decision Method Using Mobile Robot Based on Distance Between Sensor Node, International Journal of Advanced Research in Artificial Intelligence (IJARAI), Vol. 3, No.3, pp. 25-31, 2014. (http://thesai.org/Publications/ViewPaper?Volume=3\&Issue=3\&Code=IJ ARAI\&SerialNo $=5$ )

[12] K. Sawai, J. Peng and T. Suzuki: Throughput Measurement Method Using Command Packets for Mobile Robot Teleoperation Via a Wireless Sensor Network, International Journal of Advanced Computer Science and Applications (IJACSA), Vol. 7, No. 4, pp. 348-354, 2016. (http://dx.doi.org/10.14569/IJACSA.2016.070446)
[13] T. Michiba, K. Sawai, T. Oshima, K. Koyanagi, H. Masuta, T. Motoyoshi and T. Tamamoto: Development of Communication Quality Measurement Method Utilizing CMD Packet for Mobile Robot Teleoperation", Proc. of the 3rd Fukushima Research Conference on Remote Technologies for Nuclear Facilities 2018, p. 19, 2018.

[14] Y. Ichikawa, K. Sawai, P. Ju and T. Suzuki: Study of Mobile Sensor Node Deployment Method maintaining End to End Throughput in Ad Hoc Networks, Proc. of the 6th International Conference on Advanced Mechatronics (ICAM2015), p. 135, 2015.

[15] K. Sawai, S. Tanabe, R. Kunimoto, Y. Koike and T. Suzuki: Performance Evaluation of Sensor Node Deployment Strategy for Maintaining Wireless Sensor Network Communication Connectivity, Proc. of the 2012 International Symposium on Nonlinear Theory and its Applications (NOLTA2012), pp. 255-258, 2012.

[16] H. Akiyama, K. Yoshida and S. Tadokoro: Field Experiment on Multiple Mobile Robots Conducted in an Underground Mall, Field and Service Robotics Springer Tracts in Advanced Robotics, vol. 62, pp365- 375, 2010.

[17] H. Jiang, J. Qian, and W. Peng: Energy Efficient Sensor Placement for Tunnel Wireless Sensor Network in Underground Mine, Proc. of 2nd International Conference on Power Electronics and Intelligent Transportation System(PEITS 2009), pp. 219-222, 2009.

[18] J. Xu, S. Duan and M. Li: The Research of New Type Emergency Rescue Communication System in Mine Based on Wi-Fi Technology, Proc. of IEEE 3rd International Conference on Communication Software and Networks (ICCSN), pp. 8-11, 2011.

[19] K. Nagatani, S. Kiribayashi, Y. Okada, K. Otake, K. Yoshida, S. Tadokoro, T. Nishimura, T. Yoshida, E. Koyanagi, M. Fukushima and S. Kawatsuma: Emergency Response to the Nuclear Accident at the Fukushima Daiichi Nuclear Power Plants using Mobile Rescue Robots, Journal of Field Robotics, vol. 30, no. 1, pp. 44-63, 2013.

[20] Parker, E., L., Kannan, B., Xiaoquan, F., Yifan, T. (2003). Heterogeneous Mobile Sensor Net Deployment Using Robot Herding and Line of Sight Formations, Proc. of 2003 IEEE/RSJ International Conference on Intelligent Robots and Systems (IROS2003), Volume 3. pp.2488-2493, 2003. 